\title{
Nosanes $\mid$ Registros nacionais de anomalias congênitas no mundo: aspectos históricos e operacionais
}

doi: 10.1590/S1679-49742021000400015

\author{
National congenital anomaly registers in the world: historical and operational aspects
}

Registros nacionales de anomalías congénitas en el mundo: aspectos históricos y operativos

\author{
Augusto César Cardoso-dos-Santos ${ }^{1}$ - Đo orcid.org/0000-0002-1499-9105 \\ Ruanna Sandrelly de Miranda Alves' 1 - (1) orcid.org/0000-0002-2786-3207 \\ Ana Cláudia Medeiros-de-Souza' - D orcid.org/0000-0001-9317-6424 \\ João Matheus Bremm ${ }^{1}$ - @ orcid.org/0000-0002-2150-9426 \\ Julia do Amaral Gomes ${ }^{1}$ - @ orcid.org/0000-0002-0674-0494 \\ Ronaldo Fernandes Santos Alves' ${ }^{1}$ - D orcid.org/0000-0002-8358-0519 \\ Valdelaine Etelvina Miranda de Araujo' 1 - o orcid.org/0000-0003-1263-1646 \\ Giovanny Vinícius Araújo de França' - Đ orcid.org/0000-0002-7530-2017 \\ 'Ministério da Saúde, Secretaria de Vigilância em Saúde, Brasília, DF, Brasil
}

\section{Resumo}

Objetivo: Identificar registros de anomalias congênitas com cobertura nacional existentes no mundo, destacando suas principais características históricas e operacionais. Métodos: Revisão documental, mediante busca na base Medline/Pubmed e consulta a dados provenientes de relatórios, documentos oficiais e sítios eletrônicos. Foram incluídos trabalhos com relato de pelo menos um registro nacional. Resultados: Foram identificados 40 registros nacionais de anomalias congênitas em 39 países diferentes. Todos os registros incluídos no estudo localizavam-se em países de renda alta ou média superior, com concentração na Europa. A maior parte dos registros foi de base populacional, de notificação compulsória e com tempo limite para notificação de até 1 ano de idade. 0 registro brasileiro apresentou a maior cobertura anual. Conclusão: Os registros discutidos apresentaram características diversas, relacionadas à realidade de cada país. Os resultados apresentados fornecem subsídios para a temática da vigilância das anomalias congênitas, sobretudo em locais onde se deseja implementar tal atividade.

Palavras-chave: Anormalidades Congênitas; Declaração de Nascimento; Vigilância Epidemiológica; Revisão; Cooperação Internacional; Serviços de Saúde.

Correspondência:

Augusto César Cardoso-dos-Santos - Ministério da Saúde, Secretaria de Vigilância em Saúde, Unidade Técnica de Vigilância das Anomalias Congênitas, SRTVN 701, Via W5 Norte, Ed. PO700, $6^{\circ}$ andar, Brasília, DF, Brasil. CEP: 70723-040

E-mail: augusto.cesar@saude.gov.br 


\section{Introdução}

Anomalias congênitas são alterações estruturais ou funcionais que ocorrem durante a vida intrauterina. ${ }^{1}$ Além de importantes causas de óbito perinatal e neonatal, elas podem levar a incapacidades crônicas, impactando significativamente a vida dos indivíduos afetados, suas famílias e os sistemas de saúde. ${ }^{1,2}$ Segundo a Organização Mundial da Saúde, cerca de 295 mil bebês morrem nas primeiras quatro semanas de vida por conta de anomalias congênitas, a cada ano. ${ }^{1}$

A coleta sistemática e contínua de informações sobre casos de anomalias congênitas em uma população bem definida configura um registro. ${ }^{3}$ No mundo, diferentes registros de anomalias congênitas foram criados a partir da segunda metade do século XX, após o episódio conhecido como "tragédia da talidomida", quando mais de 10 mil crianças, em 46 países, nasceram com graves anomalias congênitas em decorrência do uso desse fármaco durante a gravidez. ${ }^{4} 0$ s registros de anomalias congênitas são úteis para monitorar tendências temporais ou geográficas dessas alterações, auxiliar na identificação de fatores de risco, delinear grupos populacionais vulneráveis, planejar e avaliar ofertas de cuidado à saúde, entre outras finalidades. ${ }^{3,5}$

Conhecer o perfil epidemiológico das anomalias congênitas, por meio do sistema de registro de notificação com cobertura nacional e do monitoramento com cobertura nacional, proporciona aos países a possibilidade de entender seu impacto na população e em seus sistemas de saúde. Esse conhecimento também produz informações úteis no sentido de advogar por medidas de prevenção e cuidado adequadas ao cenário específico de cada local. ${ }^{6-8}$

\section{Os registros de anomalias congênitas são úteis para monitorar tendências temporais ou geográficas dessas alterações, auxiliar na identificação de fatores de risco, delinear grupos populacionais vulneráveis, planejar e avaliar ofertas de cuidado à saúde, entre outras finalidades.}

Os registros de anomalias congênitas podem cobrir países inteiros ou uma parcela representativa da população nacional (registros nacionais), ou ainda, lugares específicos dentro de um país (registros locais ou subnacionais). Além disso, eles podem abranger todos os nascimentos de determinada área geográfica (base populacional) ou cobrir apenas nascimentos em um único hospital ou hospitais selecionados (base hospitalar).,10 Todavia, inexistem dados na literatura científica sobre 0 panorama global e atualizado dos registros nacionais de anomalias congênitas e suas particularidades.

Este trabalho teve como objetivo identificar registros de anomalias congênitas com cobertura nacional existentes no mundo, destacando suas principais características históricas e operacionais.

\section{Métodos}

Trata-se de uma nota de pesquisa de caráter documental, cuja estratégia metodológica foi detalhada em um trabalho de revisão narrativa, sobre redes internacionais para a vigilância de anomalias congênitas, anteriormente publicado. ${ }^{6} \mathrm{Em}$ resumo, para mapear e documentar os registros de anomalias congênitas com cobertura nacional existentes ao redor do mundo, buscou-se por referências bibliográficas indexadas na base Medical Literature Analysis and Retrievel System Online (Medline)/PubMed (disponibilizada pela National Library of Medicine, dos Estados Unidos) em 10 de janeiro de 2020, a partir da chave de busca especificada na Figura 1. Além dessa busca, informações relevantes, relacionadas ao tema deste trabalho, foram obtidas de relatórios, documentos oficiais e sítios eletrônicos disponibilizados pelas redes, registros e instituições que trabalham com a vigilância de anomalias congênitas.

As principais etapas metodológicas, assim como as informações extraídas de cada um dos trabalhos ou documentos encontrados, apresentam-se detalhadas na Figura 1. As informações extraídas foram analisadas por dois revisores (Cardoso-dos-Santos AC e Alves SRM), de maneira independente. Informações sobre a renda nacional bruta per capita de cada um dos países relacionados foram obtidas do relatório World Economic Situation and Prospects, elaborado pela Organização das Nações Unidas.

\section{Resultados}

Ao todo, foram identificados 40 registros de anomalias congênitas com cobertura nacional em 39 
países, cujas principais características se encontram no Quadro 1. À exceção da África, todos os continentes apresentaram pelo menos um registro nacional de anomalias congênitas. A maior parte desses países se concentrava no continente europeu, todos nas categorias socioeconômicas de renda alta (28) e média superior (12), como mostrado na Figura 2. Vinte e seis registros foram de base populacional e 12 de base hospitalar; tal informação não foi encontrada para Chile e Panamá.

0 registro nacional húngaro (Hungarian Congenital Abnormality Registry) foi o mais longevo - suas atividades oficiais iniciaram-se em 1962 - enquanto o mais recente foi o escocês, criado em 2018. A maioria dos registros nacionais (29/40) fazia parte de pelo menos uma rede internacional para vigilância de anomalias congênitas. 0 registro brasileiro foi o que apresentou maior número de nascimentos anuais - em torno de 3 milhões - e cobria
98\% dos nascimentos do país. No total, 19 registros apresentaram mais de $98 \%$ de cobertura, quase todos de base populacional; dois deles, Cuba e República Dominicana, na América Central, eram de base hospitalar.

Quatorze registros nacionais eram de caráter compulsório, e desses, 12 de base populacional; e entre os 11 de caráter voluntário, apenas cinco eram de base populacional. A idade cronológica limite para notificar um indivíduo com anomalia congênita também se revelou bem diferente entre os registros, variando desde a alta hospitalar (6) até um mês (5), ou maior ou igual a 1 ano de idade (23).

Um total de 16 registros nacionais notificavam unicamente anomalias congênitas maiores, e 14 notificavam anomalias maiores e menores. A Classificação Estatística Internacional de Doenças e Problemas Relacionados à Saúde (CID, $9^{\mathrm{a}}$ e $10^{\mathrm{a}}$ revisões) foi o principal sistema

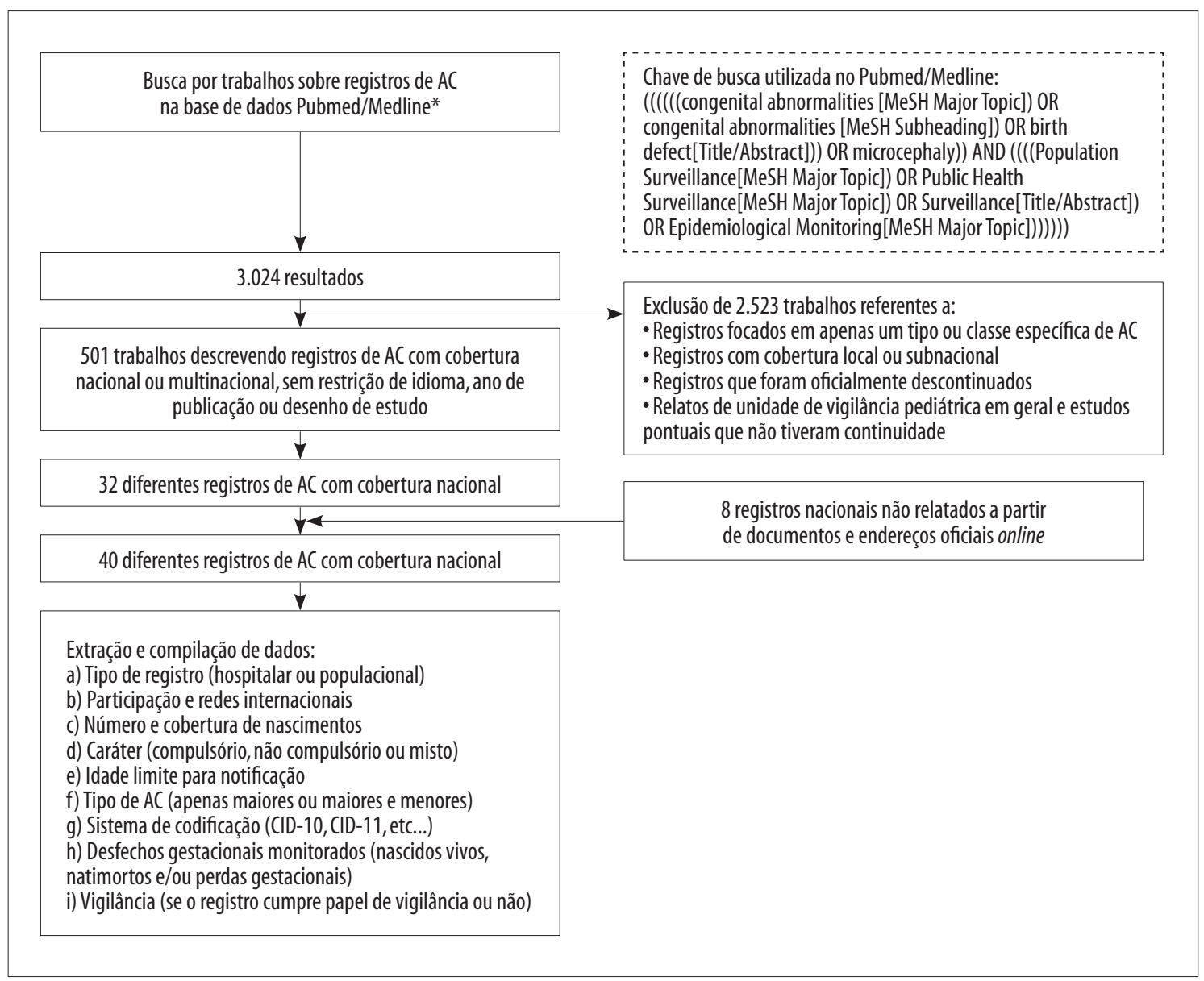

Legenda: AC:anomalias congênitas; CID:Classificação Estatística Internacional de Doenças e Problemas Relacionados à Saúde; PubMed/Medline: Medical Literature Analysis and Retrievel System Online (Medline)/PubMed, serviço disponibilizado pela National Library of Medicine dos Estados Unidos.

\section{Figura 1 - Fluxograma das principais etapas metodológicas para a identificação e seleção de registros de anomalias congênitas com cobertura nacional existentes no mundo}


Registros nacionais de anomalias congênitas

\begin{tabular}{|c|c|c|c|c|c|c|c|c|c|}
\hline 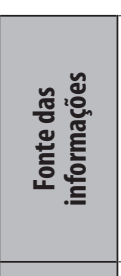 & 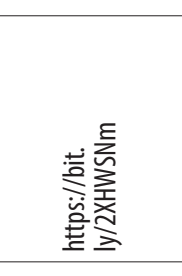 & 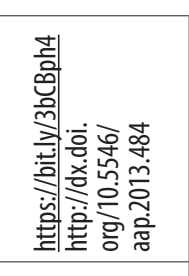 & 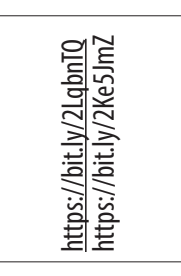 & 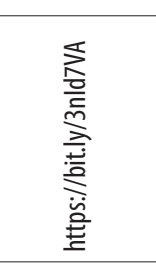 & 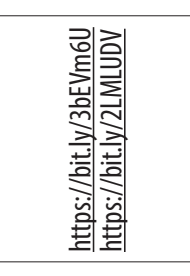 & 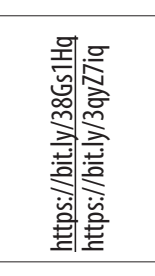 & 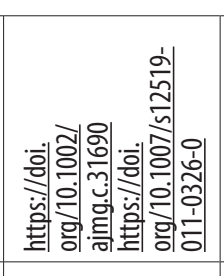 & 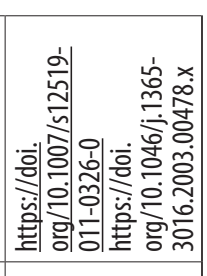 & 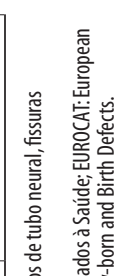 \\
\hline 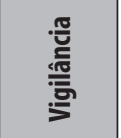 & 1 & Е & Е & $\frac{\circ}{2}$ & E & 1 & $\Xi$ & $\Xi$ & 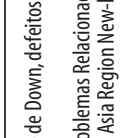 \\
\hline 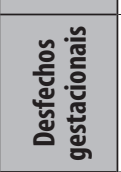 & 1 & 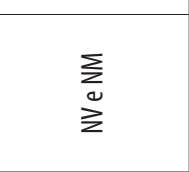 & $\begin{array}{l}\sum_{0}^{\bar{n}} \\
\sum\end{array}$ & z & $\begin{array}{l}\sum_{j}^{\bar{j}} \\
\sum\end{array}$ & I & $\begin{array}{l}\sum_{0}^{\bar{j}} \\
\sum\end{array}$ & 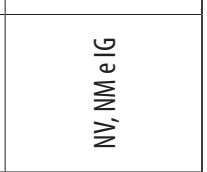 & 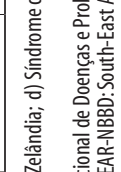 \\
\hline 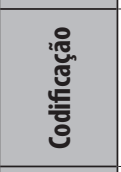 & 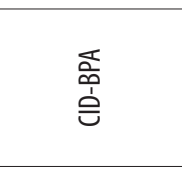 & $\begin{array}{l}\text { बे̀ } \\
\text { 仓े̀ }\end{array}$ & 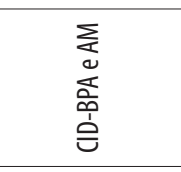 & 气 & 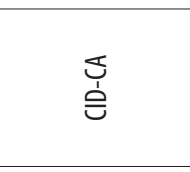 & 1 & ○ & 气 & \\
\hline 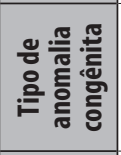 & 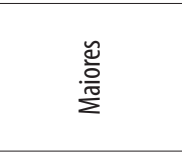 & 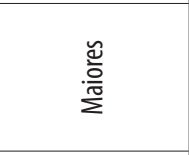 & 产 & 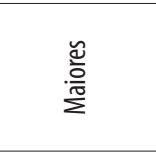 & 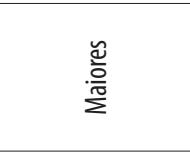 & , & 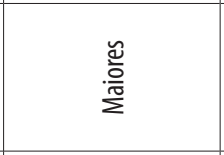 & 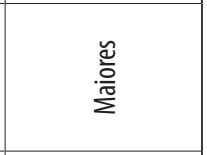 & \\
\hline 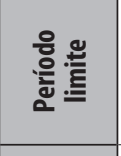 & 訔 & 焉竞 & 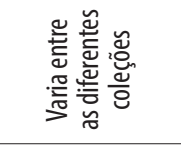 & 旁䒿 & $\stackrel{\varpi}{\Xi}$ & 1 & 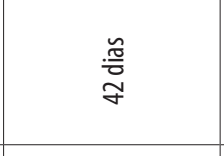 & 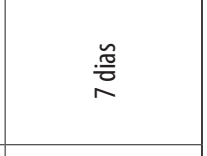 & 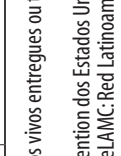 \\
\hline $\begin{array}{l}\text { 을 } \\
\frac{\text { : }}{\text { 言 }}\end{array}$ & $\Xi$ & $\stackrel{2}{2}$ & $\begin{array}{l}\text { 음 } \\
\text { 홒 }\end{array}$ & E & 1 & 1 & 1 & $\stackrel{20}{2}$ & \\
\hline 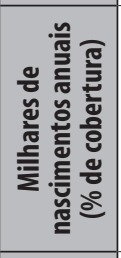 & 1 & 高 & 1 & 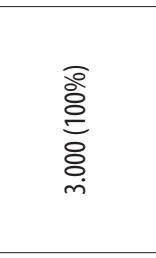 & $\begin{array}{l}\overline{\mathrm{o}} \\
\stackrel{\circ}{0} \\
\stackrel{\bar{m}}{\bar{m}}\end{array}$ & $\nexists$ & $\stackrel{\sim}{\sim}$ & 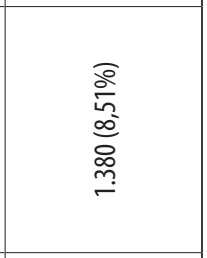 & : \\
\hline 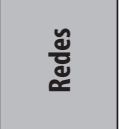 & $\begin{array}{l}\text { 前 } \\
\underline{\underline{o}}\end{array}$ & 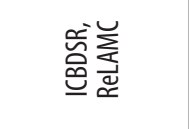 & 1 & 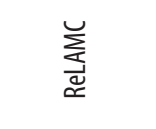 & $\begin{array}{l}\text { 楌 } \\
\underline{\underline{n}}\end{array}$ & 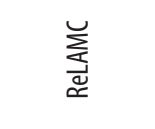 & I & I & \\
\hline 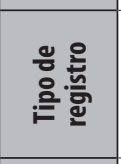 & 党 & 遌产 & 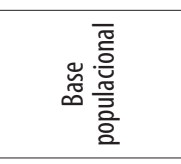 & 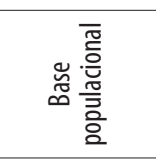 & 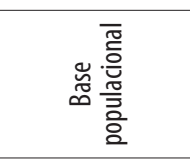 & 1 & 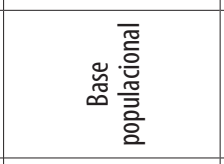 & 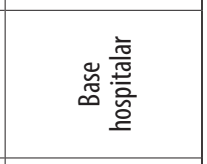 & \\
\hline 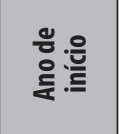 & 음 & ذ્مे & $\stackrel{\bar{g}}{\circ}$ & oे & : & ٍั & Sี & 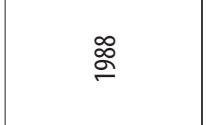 & \\
\hline 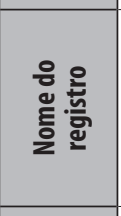 & 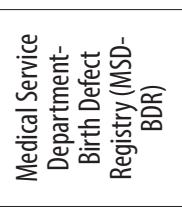 & 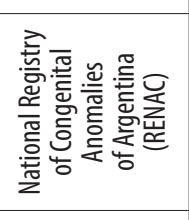 & 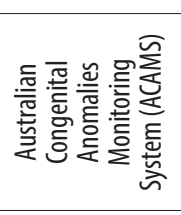 & 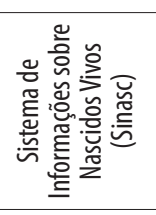 & 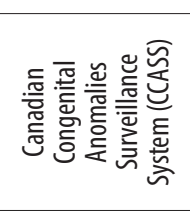 & 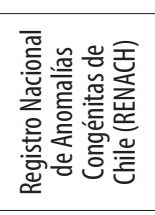 & 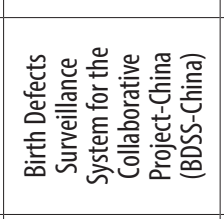 & 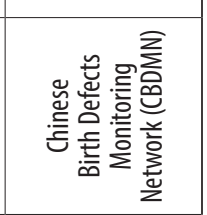 & 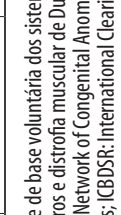 \\
\hline 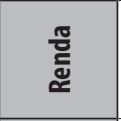 & $\frac{\pi}{\frac{\pi}{4}}$ & 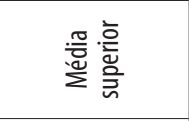 & $\frac{\pi}{4}$ & 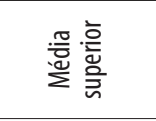 & $\frac{\pi}{\frac{\pi}{4}}$ & $\frac{\tilde{g}}{\frac{\pi}{4}}$ & 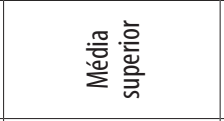 & 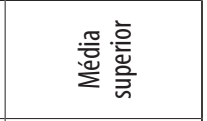 & \\
\hline$\frac{\frac{n}{\pi}}{\frac{\pi}{2}}$ & 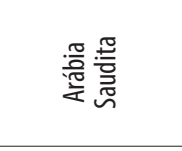 & 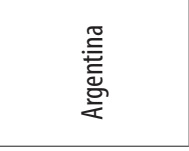 & 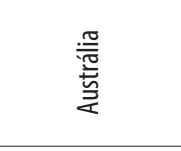 & $\overline{\overline{\overline{\check{c}}}}$ & 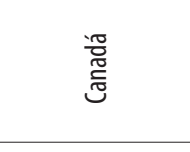 & $\frac{\underline{\nu}}{\overline{\bar{\sigma}}}$ & 䲱 & 总 & 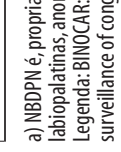 \\
\hline
\end{tabular}




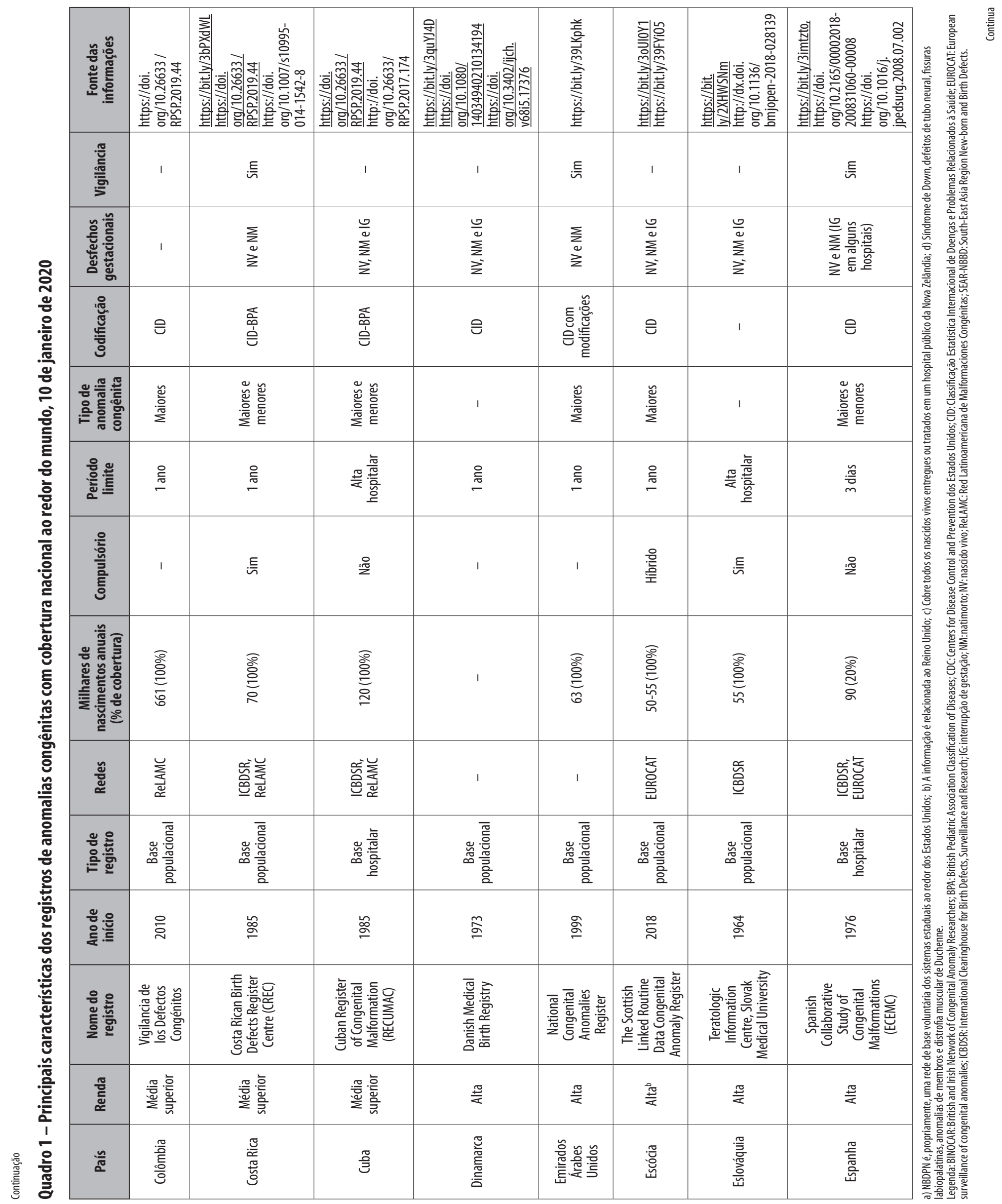




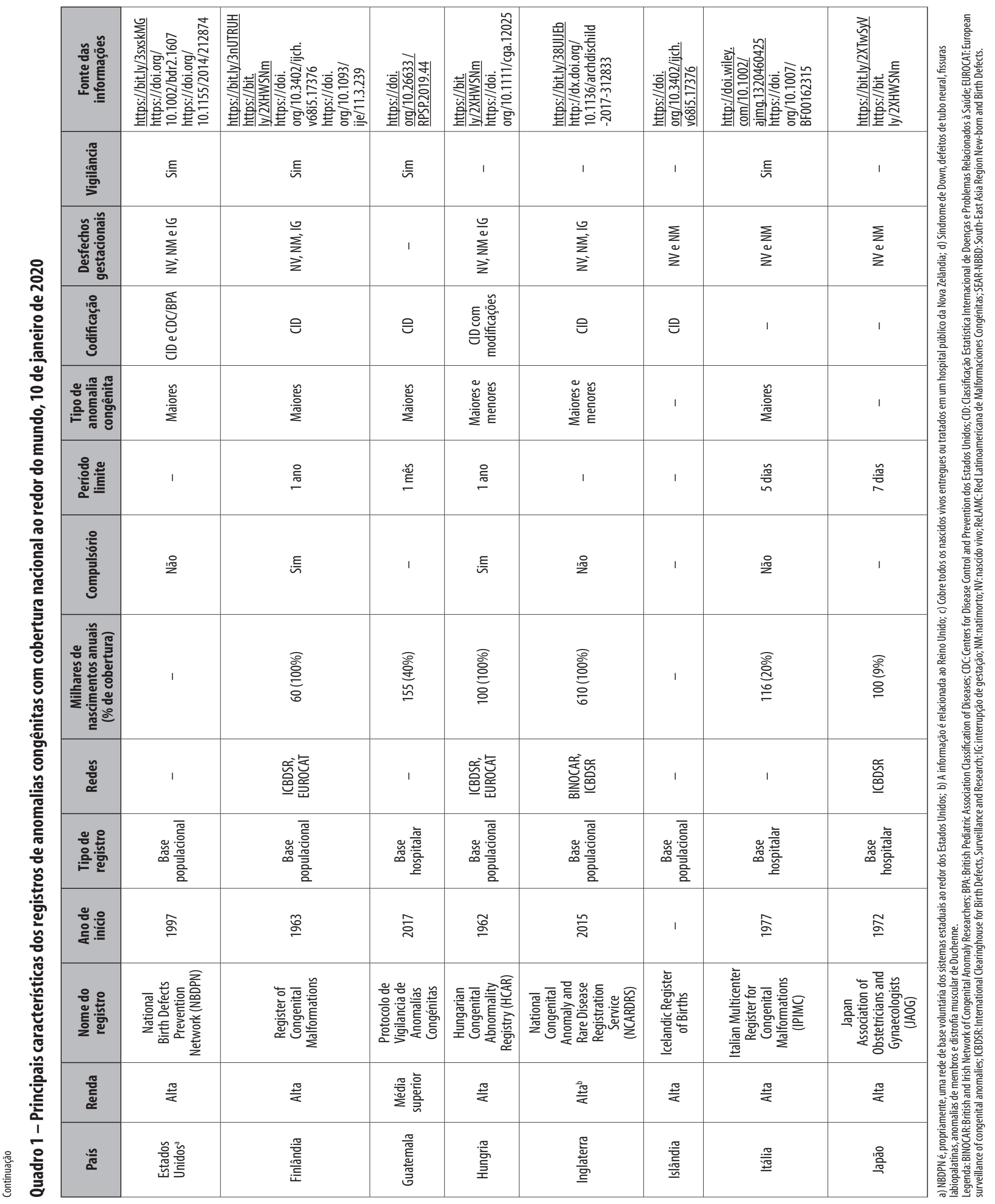




\begin{tabular}{|c|c|c|c|c|c|c|c|c|c|}
\hline & 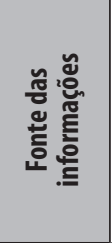 & 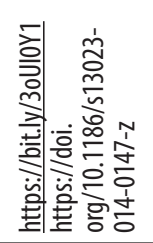 & 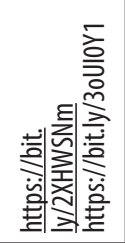 & 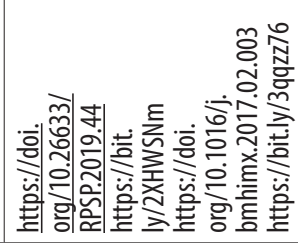 & 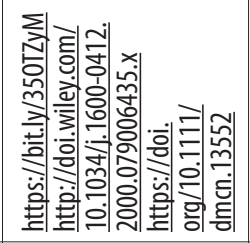 & 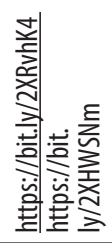 & 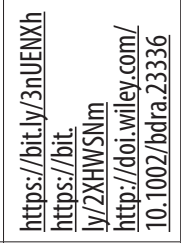 & 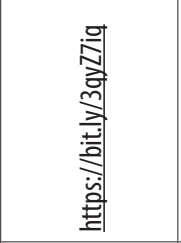 & 氛 \\
\hline & 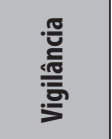 & 1 & 1 & $\Xi$ & $\Xi$ & 1 & 1 & 1 & 1 \\
\hline ஓ్ర & 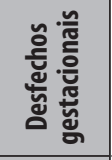 & $\sum_{\sum}^{\stackrel{0}{0}}$ & $\sum_{j}^{\infty}$ & $\sum_{\sum}^{\infty}$ & $\sum_{\sum}^{\frac{0}{N}}$ & $\sum_{i}^{\frac{O}{0}}$ & $\sum_{j}^{\frac{0}{0}}$ & 1 & 1 \\
\hline 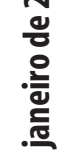 & 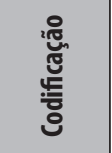 & 1 & ㅇ & 1 & $\begin{array}{l}\text { 咅 } \\
\text { 仓े }\end{array}$ & 1 & ㅇ & 1 & ○ \\
\hline $\begin{array}{l}\text { 응 } \\
\text { 음 }\end{array}$ & 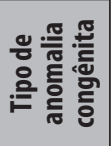 & 1 & 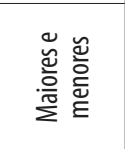 & 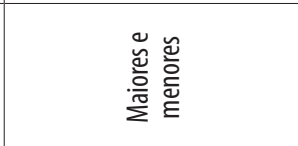 & 1 & 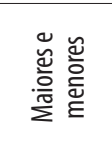 & 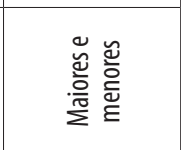 & 1 & 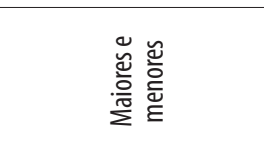 \\
\hline $\begin{array}{l}\text { 튼 } \\
\text { 흔 } \\
\text { 힌 }\end{array}$ & 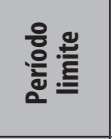 & 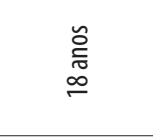 & 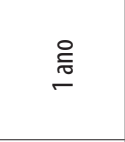 & 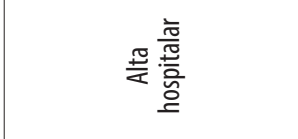 & 음 & 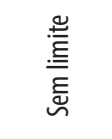 & 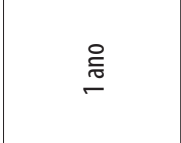 & 1 & 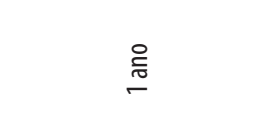 \\
\hline 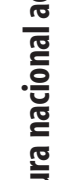 & $\begin{array}{l}\text { 은 } \\
\text { 블 } \\
\text { 흔 }\end{array}$ & E气 & $\frac{10}{2}$ & $\frac{i \pi}{2}$ & $\Xi$ & $\Xi$ & $\frac{i 0}{2}$ & 1 & $\Xi$ \\
\hline 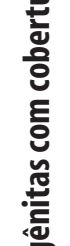 & 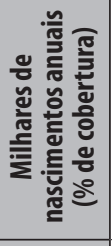 & 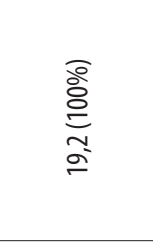 & $\frac{\text { oे }}{\stackrel{8}{\sigma}}$ & $\begin{array}{l}\text { बे } \\
\text { مे } \\
\text { స్ }\end{array}$ & $\begin{array}{l}\overline{8} \\
\text { oे } \\
\text { 응 }\end{array}$ & 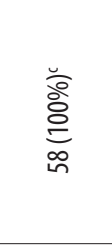 & 高 & 1 & \\
\hline 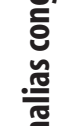 & $\frac{\check{\varpi}}{\dddot{\Xi}}$ & 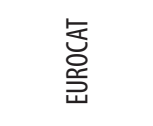 & 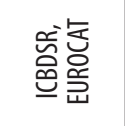 & $\begin{array}{l}\stackrel{\sim}{\tilde{O}} \\
\underline{\underline{0}}\end{array}$ & 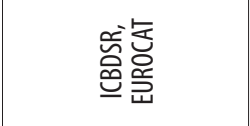 & 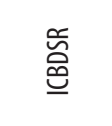 & 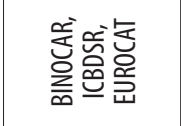 & $\sum_{\approx}^{u}$ & 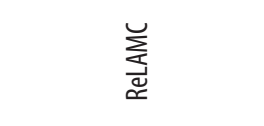 \\
\hline 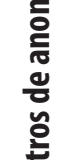 & 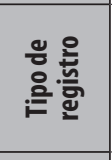 & 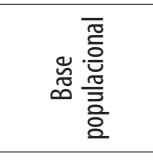 & 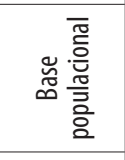 & 竞产 & 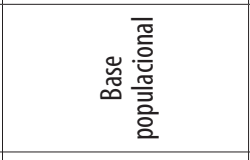 & 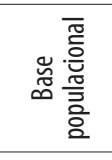 & 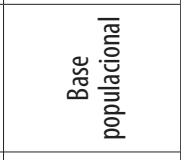 & 1 & 离 \\
\hline 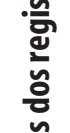 & 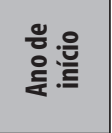 & $\stackrel{\circ}{\circ}$ & $\stackrel{\stackrel{\circ}{\circ}}{\circ}$ & $\stackrel{\infty}{\vdots}$ & & 占 & 各 & 1 & 芩 \\
\hline 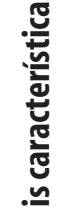 & 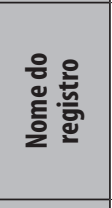 & 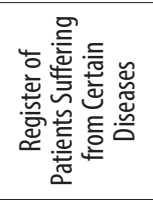 & 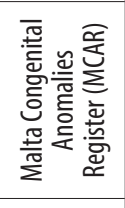 & 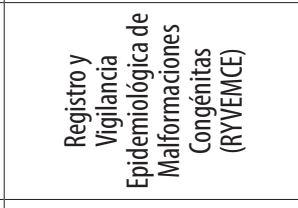 & 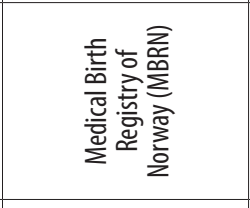 & 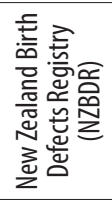 & 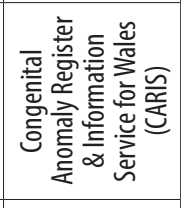 & 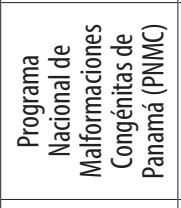 & 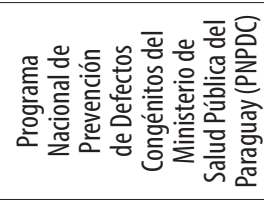 \\
\hline 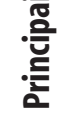 & 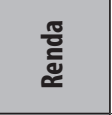 & $\frac{\text { 要 }}{4}$ & 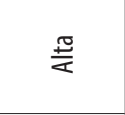 & 营䯧 & $\frac{\square}{\frac{\pi}{2}}$ & $\frac{\Gamma}{\frac{\pi}{\alpha}}$ & 尊 & $\frac{\pi}{2}$ & 穿 \\
\hline $\begin{array}{l}\frac{1}{1} \\
\frac{0}{3} \\
\frac{0}{3} \\
0\end{array}$ & $\frac{n}{\bar{c}}$ & 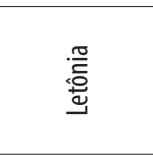 & $\frac{\frac{\pi}{5}}{\sum}$ & $\frac{\stackrel{Q}{\frac{\pi}{2}}}{\sum_{2}}$ & 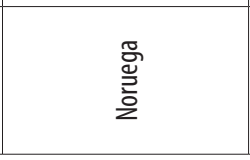 & 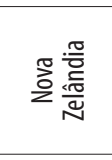 & 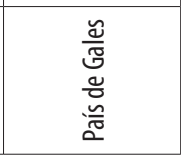 & 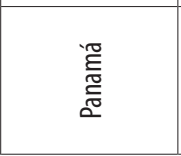 & 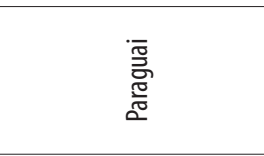 \\
\hline
\end{tabular}




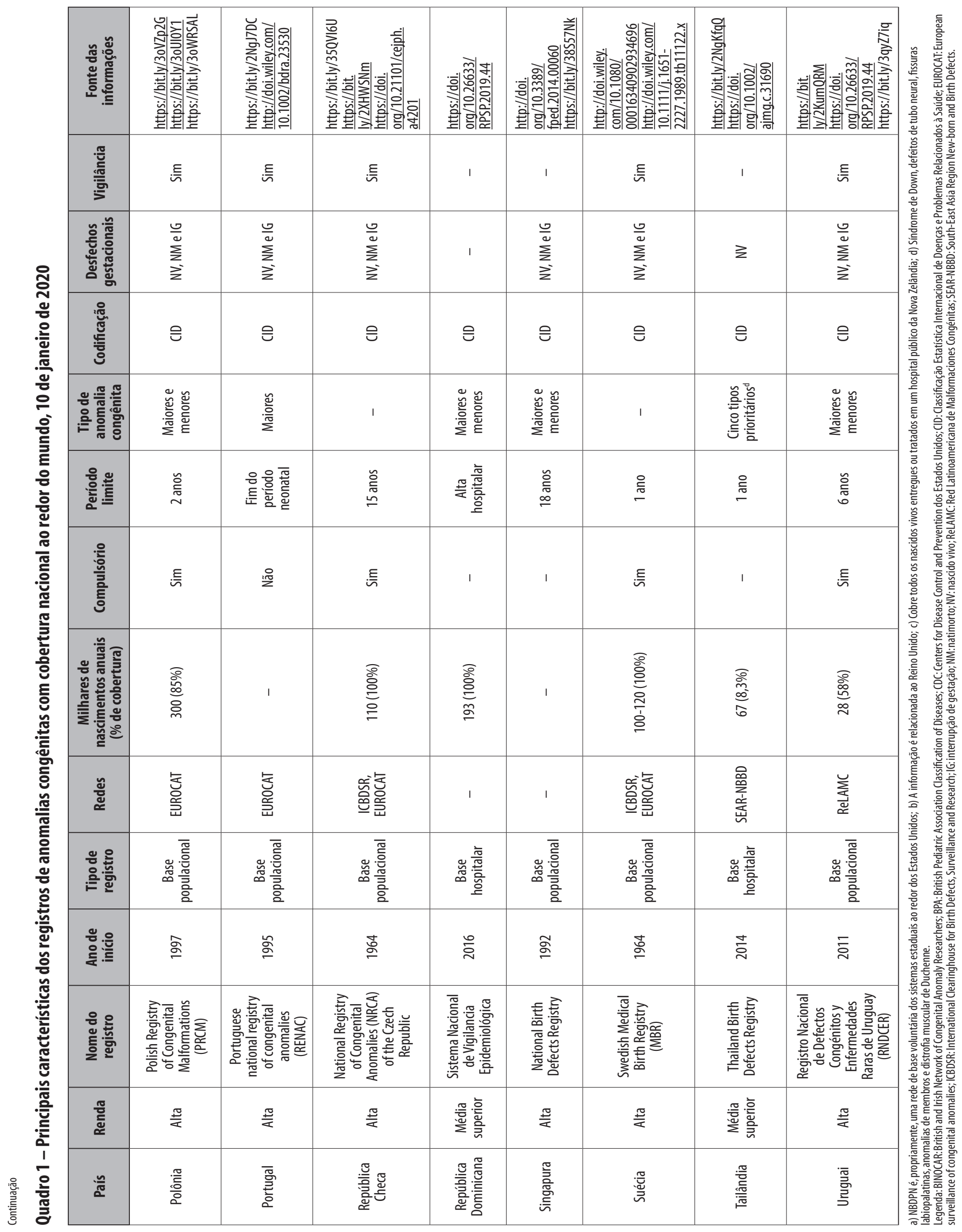




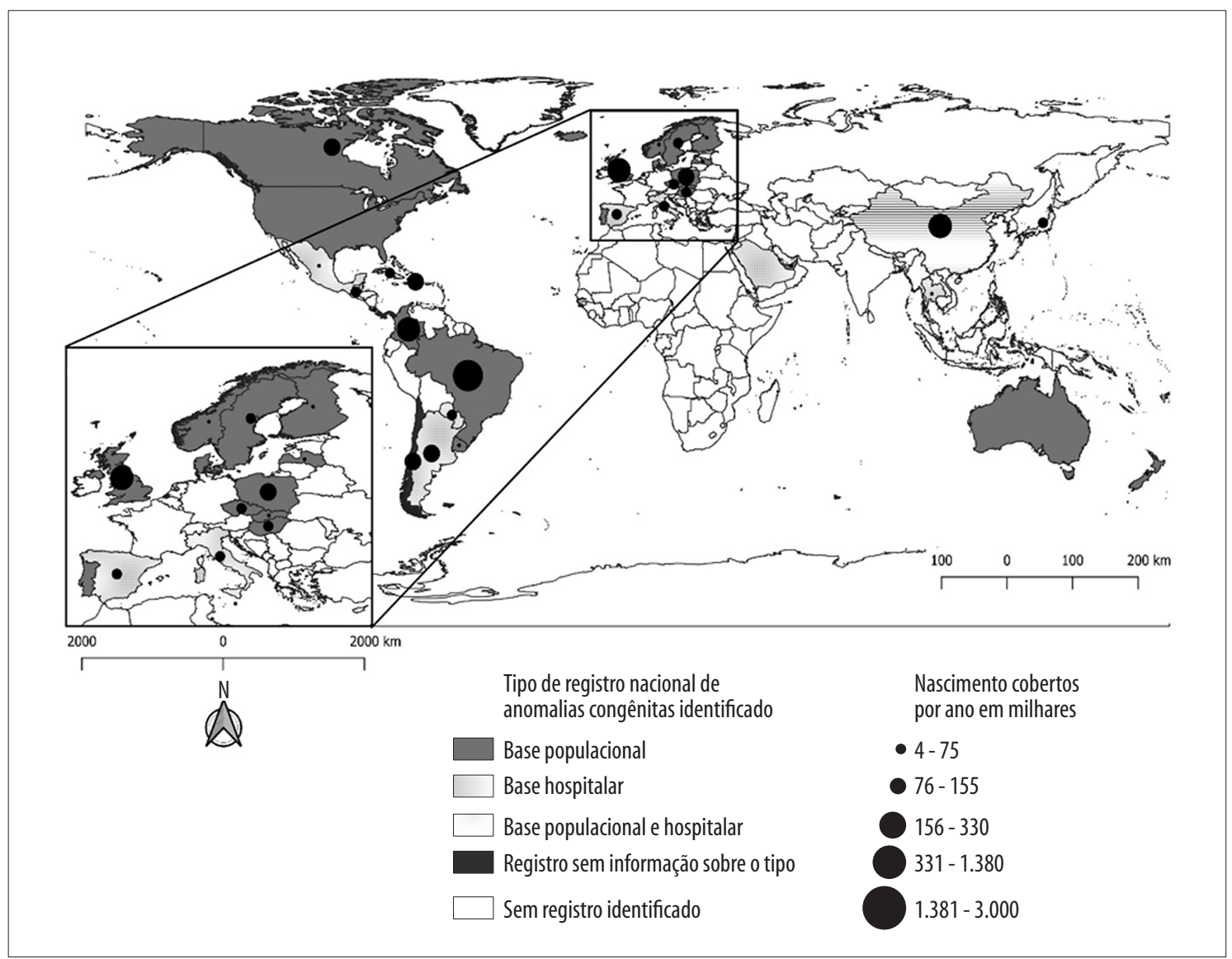

Nota: Os registros da Inglaterra, Escócia e País de Gales foram representados, em conjunto, pelo Reino Unido, e a cobertura anual foi representada pela soma dos nascimentos nos três registros de anomalias congênitas. Polígonos com preenchimento representam os países que possuem pelo menos um registro nacional de anomalias congênitas de base populacional, hospitalar ou ambas. 0 tamanho do círculo é proporcional ao número (em milhares) de nascimentos cobertos anualmente, em cada registro.

\section{Figura 2 - Distribuição mundial e cobertura anual dos registros incluídos no presente trabalho (A), com destaque para 0 continente europeu (B), 10 de janeiro de 2020}

de codificação empregado (32); porém, dez registros nacionais acrescentaram modificações à CID (sobretudo a British Pediatric Association Classification of Diseases).

Com relação aos desfechos gestacionais, 33 sistemas notificavam registros de nascidos vivos e natimortos, e desses, 20 incluíam interrupções de gestação em seu escopo. Os registros da Tailândia e do Brasil foram os únicos a incluir apenas nascidos vivos. Entre os registros pesquisados, pelo menos 19 também realizavam o processo de vigilância de anomalias congênitas em sua área de cobertura.

\section{Discussão}

Em todo o mundo, apenas 40 registros de anomalias congênitas com cobertura nacional foram encontrados em 39 diferentes países. Os registros identificados se localizavam exclusivamente em países de rendas alta e média superior, grande parte deles concentrada na Europa. Desse modo, as estimativas globais das anomalias tendem a super-representar esses lugares, em detrimento dos demais. Entretanto, trata-se de uma importante contribuição dos registros presentes nesses países a produção de uma série de materiais técnico-científicos de orientação das diferentes etapas da vigilância de anomalias congênitas.

A ausência de representatividade em países de renda média inferior e baixa é importante porque, nesses contextos, o impacto das anomalias em saúde pode ser maior, devido à baixa oferta de serviços assistenciais adequados para o cuidado de crianças afetadas. ${ }^{8}$ Além disso, nesses países, as demais 
causas de mortalidade infantil, como desnutrição, baixas condições de saneamento e suscetibilidade a infecções, dificuldade de acesso aos serviços de saúde, entre outras, ainda são comuns, o que pode ajudar a "mascarar" a real magnitude das anomalias congênitas, em termos epidemiológicos ${ }^{8,11}$

Grandes eventos, envolvendo consequências teratogênicas, como a tragédia da talidomida, ${ }^{4,12} 0$ desastre de Chernobyl ${ }^{13}$ e a epidemia de síndrome congênita associada à infecção pelo vírus Zika, ${ }^{14}$ vêm mobilizando as nações e seus territórios para o monitoramento das anomalias congênitas. A despeito do registro húngaro ser 0 mais antigo encontrado neste trabalho (1962), o programa da República Tcheca iniciou suas atividades em 1961, mas o monitoramento regular teve início em $1964 .{ }^{15}$

Os registros com cobertura nacional apresentaram diferentes características entre si. A maior parte deles foi de base populacional, o que torna as prevalências obtidas de tais sistemas menos suscetíveis a vieses, comparadas àquelas obtidas a partir de registros hospitalares. ${ }^{5,16}$ Registros de base hospitalar podem ser uma escolha interessante, sobretudo em países com registros incipientes e/ou recursos financeiros limitados; além disso, existe a possibilidade, no futuro, destes registros de anomalias se expandirem para programas de base populacional., ${ }^{5,17}$ Também é importante mencionar que alguns registros complementam suas informações por meio da busca ativa de dados, a exemplo da revisão de relatórios de alta hospitalar (caso de alguns estados dos Estados Unidos) ${ }^{18}$ auditoria perinatal (Austrália e Nova Zelândia) ${ }^{19}$ e rotinas de vinculação (linkage) entre bases de dados (Escócia) ${ }^{20}$

0 que, até quando e como notificar as anomalias congênitas também foram questões revisadas. A maior parte dos registros mostrou notificação de caráter compulsório, sobretudo de anomalias maiores de acordo com a CID, em nascidos vivos, natimortos e crianças com idade máxima notificada igual ou abaixo de 1 ano.

Embora 0 trabalho tenha investigado apenas registros com cobertura nacional, a proporção de nascimentos cobertos anualmente variou bastante. 0 registro brasileiro - Sistema de Informações sobre Nascidos Vivos (Sinasc) - foi aquele que apresentou o maior número absoluto de nascimentos cobertos anualmente.
O documento básico do Sinasc é a Declaração de Nascido Vivo, na qual, desde 1999, constam dados sobre a presença e 0 tipo de anomalia congênita. ${ }^{21,22}$

Apesar de a vigilância hospitalar ser realizada em alguns poucos hospitais do Brasil, por meio do Estudo Colaborativo Latino-Americano de Malformações Congênitas (ECLAMC), a vigilância de anomalias congênitas ainda não acontece sistematicamente, em todo o território nacional. Entretanto, um modelo de vigilância nacional vem sendo estruturado na Secretaria de Vigilância em Saúde do Ministério da Saúde brasileiro, em articulação com demais secretarias do órgão, membros da comunidade acadêmica e sociedades médicas. ${ }^{23-25}$

Este trabalho apresentou algumas limitações, como a possibilidade de haver registros com cobertura nacional não capturados pela abordagem metodológica utilizada, devido, por exemplo, à inexistência de material publicado online. Ademais, os registros são dinâmicos e características como a cobertura podem mudar ao longo do tempo.

Em conclusão, este trabalho revisou os principais registros de anomalias congênitas com cobertura nacional ao redor do mundo. $0 \mathrm{~s} 40$ registros analisados apresentaram diferentes características, constituindo-se em material de consulta para profissionais interessados pela temática e, sobretudo, fornecendo subsídios à reflexão sobre atividades de vigilância e quais características são desejáveis ou possíveis de serem implementadas, considerando-se a realidade de cada nação.

\section{Contribuição dos autores}

Cardoso-dos-Santos AC, Alves RSM, Medeiros-de-Souza AC, Bremm JM, Gomes JA, Alves RFS, Araujo VEM e França GVA participaram da concepção, delineamento do estudo, redação ou revisão crítica relevante do conteúdo intelectual do manuscrito. Cardoso-dos-Santos AC, Alves RSM e França GVA participaram da análise e interpretação dos dados. Todos os autores aprovaram a versão final desta nota de pesquisa e assumem responsabilidade por todos os aspectos do trabalho, incluindo a garantia de sua precisão e integridade. 


\section{Referências}

1. World Health Organization. Congenital anomalies [Internet]. Geneva: WHO; 2020 [acesso 28 maio 2020]. Disponível em: https://www.who.int/healthtopics/congenital-anomalies\#tab=tab_1

2. Thong M-K. Birth defects registries in the genomics era: challenges and opportunities for developing countries. Front Pediatr. 2014 Jun 16;2:60. doi: https://doi.org/10.3389/fped.2014.00060.

3. Lechat MF, Dolk H. Registries of congenital anomalies: EUROCAT. Environ Health Perspect. 1993;101(suppl

2):153-7. doi: http://doi.org/10.1289/ ehp.93101s 2153

4. Moro A, Invernizzi N. A tragédia da talidomida: a luta pelos direitos das vítimas e por melhor regulação de medicamentos. Hist Cienc Saude Manguinhos. 2017;24(3):603-22. doi: http://doi.org/10.1590/ S0104-59702017000300004.

5. World Health Organization. Birth defects surveillance: a manual for programme managers. Geneva: HOW; 2014.

6. Cardoso-dos-Santos AC, Magalhães VS, Medeirosde-Souza AC, Bremm JM, Alves RFS, Araujo VEM, et al. International collaboration networks for the surveillance of congenital anomalies: a narrative review. Epidemiol Serv Saude. 2020;29(4):e2020093. doi: https://doi.org/10.5123/S167949742020000400003.

7. United Nations. World Economic situation and prospects. New York; UN; 2020 [acesso 21 jul. 2020]. Disponível em: https://www.un.org/development/desa/ dpad/wp-content/uploads/sites/45/WESP2020_Annex. pdf

8. Penchaszadeh VB. Preventing congenital anomalies in developing countries. Community Genet. 2002;5(1):61-9. doi: https://doi. org/10.1159/000064632.

9. Miller JR, Lowry RB. Birth defects registries and surveillance. In: Wilson JG, Fraser FC, editors. Comparative, maternal, and epidemiologic aspects. Boston, MA: Springer; 1977 [acesso 22 jul. 2020]. doi: http://doi.org/10.1007/978-1-4615-8936-5_8.

10. Centers for Disease Control and Prevention. Birth defects surveillance course overview [Internet]. Atlanta: CDC; 2019 [acesso 23 Jul 2020]. Disponível em: https://www.cdc.gov/ncbddd/birthdefects/ surveillancemanual/facilitators-guide/courseoverview.html
11. Victora CG, Aquino EM, Leal MC, Monteiro CA, Barros FC, Szwarcwald CL. Maternal and child health in Brazil: progress and challenges. Lancet. 2011 May 28;377(9780):1863-76. doi: http://doi.org/10.1016/ S0140-6736(11)60138-4.

12. Weatherall JAC, Haskey JC. Surveillance of malformations. Br Med Bull. 1976;32(1):39-44. doi: https://doi.org/10.1186/s12884-019-2542-X.

13. Wertelecki W. Birth defects surveillance in Ukraine: a process. J Appl Genet. 2006;47(2):143-9. doi: http:// doi.org/10.1007/BF03194614.

14. Ministério da Saúde (BR) Síndrome congênita associada à infecção pelo vírus Zika. Bol Epidemiol. 2020;51(47):1-31.

15. Kucêra MJ. Congenital malformation surveillance systems. Int J Epidemiol. 1986;15(3):430. doi: https://doi.org/10.1093/ije/15.3.430.

16. Dai L, Zhu J, Liang J, Wang Y-P, Wang H, Mao M. Birth defects surveillance in China. World J Pediatr. 2011;7(4):302-10. doi: https://doi.org/10.1007/ s12519-011-0326-0.

17. Durán P, Liascovich R, Barbero P, Bidondo MP, Groisman B, Serruya S, et al. Sistemas de vigilancia de anomalías congénitas en América Latina y el Caribe: presente y futuro. Rev Panam Salud Publica. 2019;43:e44. doi: http://doi.org/10.26633/ rpsp.2019.44.

18. Centers for Disease Controle and Prevention. Statebased birth defects tracking systems [Internet]. Atlanta: CDC; 2020 [acesso 28 maio 2021]. Disponível em: https://www.cdc.gov/ncbddd/ birthdefects/states/index.html

19. Australian \& New Zealand Neonatal Network (ANZNN) [Internet]. Sydney: NPESU; 2013 [acesso 28 maio 2021]. Disponível em: https://npesu.unsw.edu.au/ data-collection/australian-new-zealand-neonatalnetwork-anznn

20. European Commission. Scottish linked routine data congenital anomaly register [Internet]. 2020 [acesso 23 jul. 2020]. Disponível em: https://eu-rd-platform. jrc.ec.europa.eu/eurocat/eurocat-members/registries/ scottish_en

21. Brasil. Lei n. 13.693/18 de 10 de julho de 2018, . Institui o dia nacional de doenças raras [Internet]. Brasília, DF: Diário Oficial da União [acesso 19 fev. 2020]. Disponível em: https://presrepublica.jusbrasil. com.br/legislacao/599358970/lei-13693-18 
22. Luquetti DV, Koifman RJ. Surveillance of birth defects: Brazil and the US. Cien Saude Colet. 2011;16(suppl 1):777-85. doi: https://doi.org/10.1590/\$141381232011000700008 .

23. Ministério da Saúde (BR). Anomalias congênitas no Brasil, 2010 a 2018: análise dos dados de sistemas de informação para o fortalecimento da vigilância e atenção em saúde. Bol Epidemil. 2021 [acesso 10 jul. 2020];52(6):1-22. Disponível em: https://bit. ly/32S8f8h

24. Cardoso-dos-Santos AC, Medeiros-de-Souza AC, Bremm JM, Alves RFS, Araújo VEM, Leite JCL, et al.

\footnotetext{
Abstract

Objective: To identify registers of congenital anomalies with national coverage currently available around the world, highlighting their main historical and operational characteristics. Methods: This was a documentary study by means of a Medline database search (via PubMed) and searches involving reports, official documents and websites. Studies reporting at least one national registry were included. Results: 40 registers of national congenital anomalies were identified in 39 different countries. All registers included in the study were concentrated in upper-middle or high-income countries located in Europe. Most of the registers were population-based, compulsory notification and with a time limit for notification of up to 1 year of age. The Brazilian register showed the highest annual coverage. Conclusion: The registers analyzed showed different characteristics, related to the reality of each country. The results presented provide support for the theme of congenital anomalies surveillance, especially in places where such activity is intended to be implemented.

Keywords: Congenital anomalies; Birth Declaration; Epidemiological Monitoring; Review; International Cooperation; Health Services.
}

List of priority congenital anomalies for surveillance under the Brazilian Information system on live births. Epidemiol Serv Saude. 2021;30(1):e2020835. doi: http://doi.org/10.1590/s1679-49742021000100030.

25. Ministério da Saúde (BR). Saúde Brasil 2018: uma análise da situação de saúde e das doenças e agravos crônicos: desafios e perspectivas [Internet]. Brasília, DF: MS; 2019 [acesso 10 jul. 2020]. Disponível em: https://bvsms.saude.gov.br/bvs/publicacoes/saude_ brasil_2018_analise_situacao_saude_doencas_ agravos_cronicos_desafios_perspectivas.pdf

\section{Resumen}

objetivo: Identificar registros de anomalias congénitas con cobertura nacional existentes en el mundo, destacando sus principales características históricas y operativas. Métodos: Revisión documental de literatura en la base de datos Medline/Pubmed y datos de informes, documentos oficiales y sitios web. Se incluyeron trabajos con informes de al menos un registro nacional. Resultados: Se identificaron 40 registros nacionales de anomalias congénitas en 39 países diferentes. Todos los registros incluidos se ubicaron en países de ingresos altos y medianos altos, con una concentración en Europa. La mayoría de los registros eran de base poblacional, con notificación obligatoria y un límite de tiempo de notificación de hasta 1 año. El registro brasileño presentá la cobertura anual más alta. Conclusión: Los registros discutidos presentaban características diferentes $y$ relacionadas con la realidad de cada país. Los resultados presentados proporcionan subsidios para la vigilancia de anomalías congénitas, especialmente en lugares que deseen implementar dicha actividad.

Palabras-clave: Anomalias Congénitas; Certificado de Nacimiento; Monitoreo Epidemiológico; Revisión; Cooperación Internacional; Servicios de Salud.

Recebido em 04/02/2021

Aprovado em 24/05/2021 